\title{
Comparison of Face Washing and Face Wiping Methods for Trachoma Control: A Pilot Study
}

\begin{abstract}
Alexandra Czerniewska, ${ }^{1}$ Aalbertus Versteeg, ${ }^{2}$ Oumer Shafi, ${ }^{2,3}$ Gebeyehu Dumessa, ${ }^{3}$ Muluadam Abraham Aga, ${ }^{3}$ Anna Last, ${ }^{2}$ David MacLeod, ${ }^{4}$ Virginia Sarah, ${ }^{5}$ Sarity Dodson, ${ }^{6}$ Nebiyu Negussu, ${ }^{7}$ Birhanu Kenate Sori, ${ }^{8}$ Michael Kirumba, ${ }^{3}$ Adam Biran, ${ }^{1}$ Sandy Cairncross, ${ }^{1}$ Matthew J. Burton, ${ }^{9,10}$ and Katie Greenland ${ }^{1 *}$

${ }^{1}$ Department of Disease Control, London School of Hygiene and Tropical Medicine, London, United Kingdom; ${ }^{2}$ Clinical Research Department, London School of Hygiene and Tropical Medicine, London, United Kingdom; ${ }^{3}$ The Fred Hollows Foundation, Addis Ababa, Ethiopia; ${ }^{4}$ Department of Infectious Disease Epidemiology, London School of Hygiene and Tropical Medicine, London, United Kingdom; ${ }^{5}$ The Fred Hollows Foundation, London, United Kingdom; ${ }^{6}$ The Fred Hollows Foundation, Sydney, Australia; ${ }^{7}$ Federal Ministry of Health, Addis Ababa, Ethiopia; ${ }^{8}$ Oromia Regional Health Bureau, Addis Ababa, Ethiopia; ${ }^{9}$ Clinical Research Department, International Centre for Eye Health, London School of Hygiene and Tropical Medicine, London, United Kingdom; ${ }^{10}$ Cornea and External Eye Department, Moorfields Eye Hospital NHS Trust, London, United Kingdom
\end{abstract}

Abstract. Eye-to-eye transmission of Chlamydia trachomatis, the causative agent of trachoma, may be plausibly interrupted if faces are kept free of ocular and nasal discharge. Between April and June 2018, 83 children aged 1-9 years with active trachoma were recruited from 62 households and allocated to a face cleaning protocol: face washing with water, face washing with water and soap, or face wiping. Faces were examined for the presence of ocular and nasal discharge, and swabs were taken from faces and hands to test for $C$. trachomatis at baseline, immediately post protocol, and after 1, 2, and 4 hours (washing protocols). Washing with soap was more effective at removing ocular discharge than either washing with water ( $89 \%$ and $27 \%$ of discharge removed, respectively, $P=0.003)$ or wiping with a hand (42\%, $P=0.013)$. The reduction in prevalence of ocular discharge was sustained for at least four hours. The prevalence of C. trachomatis on face swabs was reduced by all washing protocols. The importance of soap should not be overlooked during facial cleanliness promotion.

\section{INTRODUCTION}

Trachoma is the most common infectious cause of blindness worldwide. ${ }^{1}$ Children are the main reservoir of ocular infection with the bacterium Chlamydia trachomatis. This provokes chronic conjunctival inflammation, scarring, and inturned eyelids, which can lead to irreversible corneal damage and sight loss.

Endemic trachoma is maintained through ongoing transmission. Improving facial cleaning practice across at-risk communities is a key pillar of the WHO's strategy for trachoma elimination. ${ }^{1}$ Chlamydia trachomatis is probably transmitted through the transfer of ocular and nasal discharge of an infected person. Hypothesized C. trachomatis transmission routes, such as via the face or hands, contaminated objects, or mechanical vectors, are plausibly interrupted if faces are kept free of discharge. ${ }^{1,2}$

Although research has linked prevalence of disease symptoms with facial cleanliness, ${ }^{3}$ there are limited systematic data on the effectiveness of different face cleaning techniques or frequencies to reduce the risk of transmission. ${ }^{4}$ Health promotion programs consequently recommend an array of face washing messages and times. ${ }^{4}$ Most recommend soap use, but emphasis varies. The current study, conducted to inform the design of a large-scale face washing intervention, aimed to identify the most effective hygiene technique and frequency to minimize potential $C$. trachomatis transmission.

\section{METHODS}

We conducted this study in the West Arsi Zone of Oromia, Ethiopia, between April and June 2018. The study was nested within a cross-sectional study conducted in 247 randomly selected households with at least one child aged 1-9 years with clinically diagnosed trachomatous inflammation,

* Address correspondence to Katie Greenland, London School of Hygiene and Tropical Medicine, Keppel St., London WC1E 7HT, United Kingdom. E-mail: katie.greenland@Ishtm.ac.uk follicular (TF) or trachomatous inflammation, intense (TI). ${ }^{5,6}$ Children aged 1-9 years with TF/TI were eligible for inclusion in this sub-study.

We defined ocular discharge as the presence of clear or cloudy fluid, or dry matter, on the lid margin or lid (including the corners) and nasal discharge as the presence of wet or dry discharge visible outside the nostril nares. ${ }^{7}$ At baseline, faces were examined for the presence of ocular and nasal discharge. A trained field-worker collected sterile, dacron swab samples from 1) the eye (to determine C. trachomatis infection status), 2) the face, and 3) the hands. As per the Standard Operating Procedure, ocular swabs were wiped four times across the (everted) left upper tarsal conjunctival surface. Face and hand swabs were premoistened with sucrose phosphate buffer (2SP) and systematically rubbed with moderate, consistent pressure, tracing a line under the right eye, across the right cheek and under the nare of the right nostril (face), or across palms and backs of both hands, finger pads, and in between each finger (hands). One air control swab was randomly collected for each 50 samples to evaluate field and laboratory contamination. Gloves were changed between swabs. Swabs were stored immediately in microtubes containing $500 \mu \mathrm{L}$ of 2SP transport medium on ice packs, transferred to a $-20^{\circ} \mathrm{C}$ freezer within 8 hours of collection and then to $a-80^{\circ} \mathrm{C}$ freezer within 1 week, until testing.

Eligible children were allocated to one of four face cleaning protocols on an alternating basis as recruited with a view to including 20 children in each group. For pragmatic reasons, as multiple teams were operating in the field, eligible children in households recruited in the morning were allocated alternately to Protocols 1 and 2, and those from households recruited in the afternoons were allocated alternately to Protocols 3 and 4. All eligible children in a household were allocated to the same protocol. The protocols are given as follows: 1 ) the face was washed with water only ("washed with water"), 2) the face was washed with soap and water ("washed with soap"), or the face was wiped by the caregivers' hand ("wiped with hand") followed by handwashing for up to 30 seconds with water (Protocol 3) or water and soap (Protocol 4) (Figure 1). 

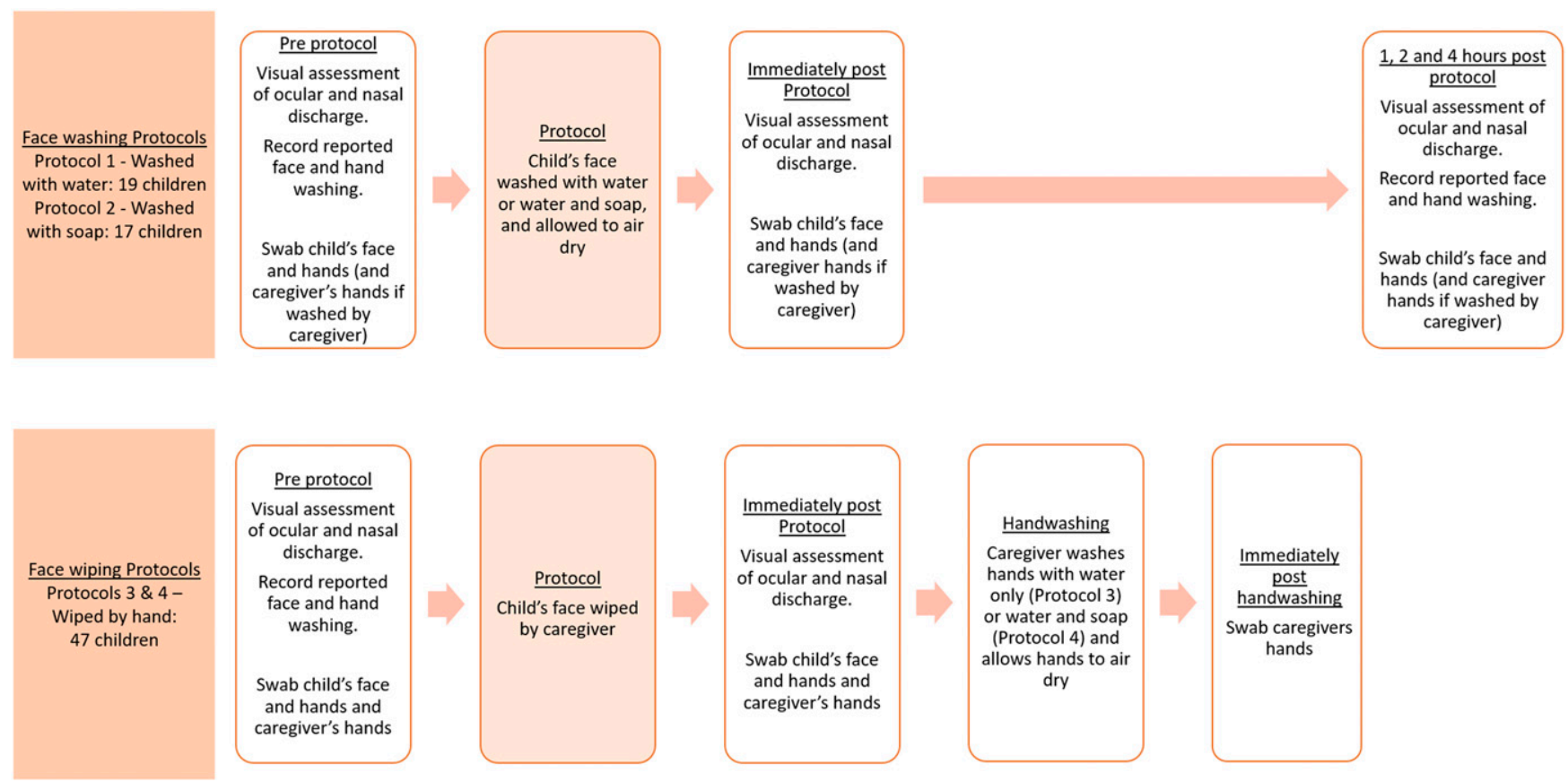

FIGURE 1. Schematic illustrating the face washing and face wiping protocols. This figure appears in color at www.ajtmh.org.

Protocols 3 and 4 were grouped together for analysis in this study. Face washing aimed to simulate naturalistic washes and was, thus, performed by the caregiver or the child, whichever behavior was reported as normal. Face washing technique was demonstrated and involved both hands rubbing the whole face including around the eyes for about 30 seconds, irrespective of soap use. Face wiping involved the caregiver using their hand(s) to remove visible discharge from the eyes and nose.

Children's faces and relevant hands were examined and swabbed again (once dried) after completing the allocated protocol (immediately post-protocol), following the aforementioned procedure. Examinations and swabbing were repeated 1, 2, and 4 hours after washing protocols. Hands of caregivers of children allocated to a wiping protocol were swabbed again after handwashing (not reported). Study personnel performed all examinations and, thus, were not masked to protocol allocation.

DNA was extracted from the swabs using a commercially available kit (Biochain Blood and Serum kit, AMS Biotechnology Europe Ltd., Abingdon, UK) and tested using a previously described multiplex real-time quantitative polymerase chain reaction (qPCR) assay ${ }^{6,8}$ to determine the presence of $C$. trachomatis. Samples were classified as C. trachomatis positive if amplification of the omcB (chromosomal) or pORF2 (plasmid) target was detected by qPCR in any well within 40 cycles. We performed chi-squared tests of association for the presence of discharge at each time point measured, among those with discharge before washing.

\section{RESULTS}

We recruited 83 children aged $1-9$ years (median age $=4$; range, 1-9) with TF/TI from 62 households. Ocular C. trachomatis was detected in 11 (13\%) children. Participants were from Muslim households where the main occupation was subsistence farming. Households had no access to piped water on the premises, and either an "unimproved" pit latrine or no latrine. Almost all children (80 children; 96\%) reported face washing in the morning before the study, but only three (4\%) had used soap. At baseline, 57 children (69\%) had ocular discharge and 65 (78\%) had nasal discharge.

We found significant differences in the presence of ocular discharge post-protocol (Table 1). Washing with soap was more effective at removing visible signs of ocular discharge immediately post-protocol than either washing with water (89\% and $27 \%$ of discharge removed, respectively, $P=$ $0.003)$, or wiping with a hand $(42 \%, P=0.013)$. No evidence was found of a difference between washing with water and wiping with a hand at removing visible signs of ocular discharge $(P=0.265)$ at this time point.

Figure 2 shows how the presence of ocular and nasal discharge changed over the 4-hour follow-up. The most pronounced and sustained reduction in discharge was observed for ocular discharge in the "washed with soap" group (Figure 2A). The removal of nasal discharge by either washing method was limited and not sustained overtime (Figure 2B).

We found $C$. trachomatis on the faces of 13 children at baseline (16\%), including two children without ocular C. trachomatis infection. Face cleaning removed $C$. trachomatis from one of three $(33 \%)$ children washed with water, one of $1(100 \%)$ child washed with soap, and three of nine (33\%) children whose faces were wiped by the hands (Supplemental Table 1). Wiping transferred C. trachomatis to the caregiver's hand without removing it from the child's face on five of six occasions. No C. trachomatis was detected on air control swabs.

\section{DISCUSSION}

In this study of three facial cleaning methods, we found evidence that ocular discharge was removed most effectively 
TABLE 1

Ocular and nasal discharge immediately post-protocol by the removal type, among those with ocular discharge present at baseline

\begin{tabular}{|c|c|c|c|c|}
\hline Removal type & N & Discharge present at baseline, $N(\%)$ & $\begin{array}{c}\text { Discharge present following protocol, } \\
N \text { (\% among those with discharge at baseline) }\end{array}$ & $P$-value ${ }^{*}$ \\
\hline \multicolumn{5}{|l|}{ Ocular discharge } \\
\hline Washed with soap & 17 & $9(53)$ & $1(11)$ & 0.013 \\
\hline Washed with water & 19 & $15(79)$ & $11(73)$ & \\
\hline Wiped with hand & 47 & $33(70)$ & $19(58)$ & \\
\hline \multicolumn{5}{|l|}{ Nasal discharge } \\
\hline Washed with soap & 17 & $11(65)$ & $3(27)$ & \\
\hline Washed with water & 19 & $14(74)$ & $7(50)$ & 0.265 \\
\hline Wiped with hand & 47 & $40(85)$ & 7 (18) & \\
\hline
\end{tabular}

by washing with soap and water, and that the reduction in prevalence of ocular discharge was sustained for at least 4 hours. Comparatively, ocular discharge was removed less effectively by washing with water alone or by a caregiver wiping discharge from a child's face with their hands. We found no significant difference between wiping and washing with water to remove ocular discharge, but wiping requires an extra step ("wiper" removing discharge transferred to their hands) to prevent onward transmission. We found no evidence that nasal discharge was removed effectively by any of the three methods.

Our finding that ocular discharge takes hours to return after washing with soap is consistent with the findings from the

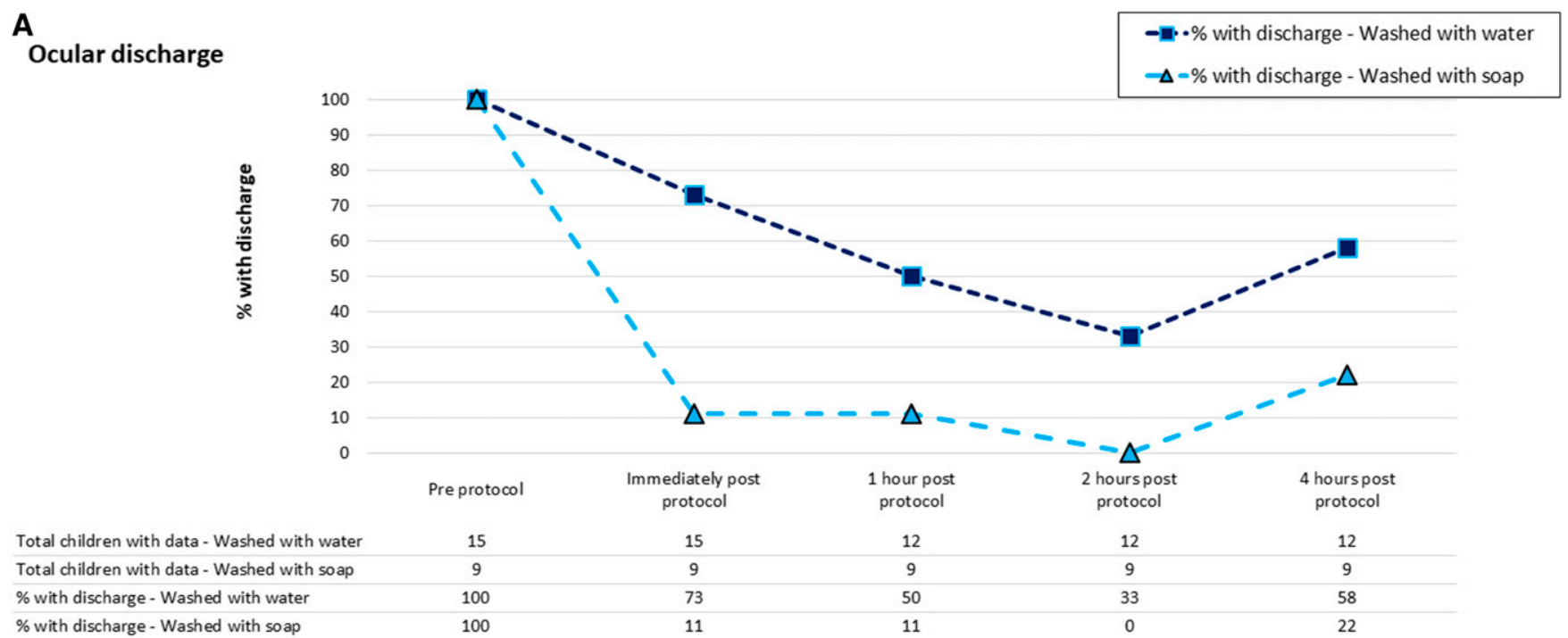

B

\section{Nasal discharge}

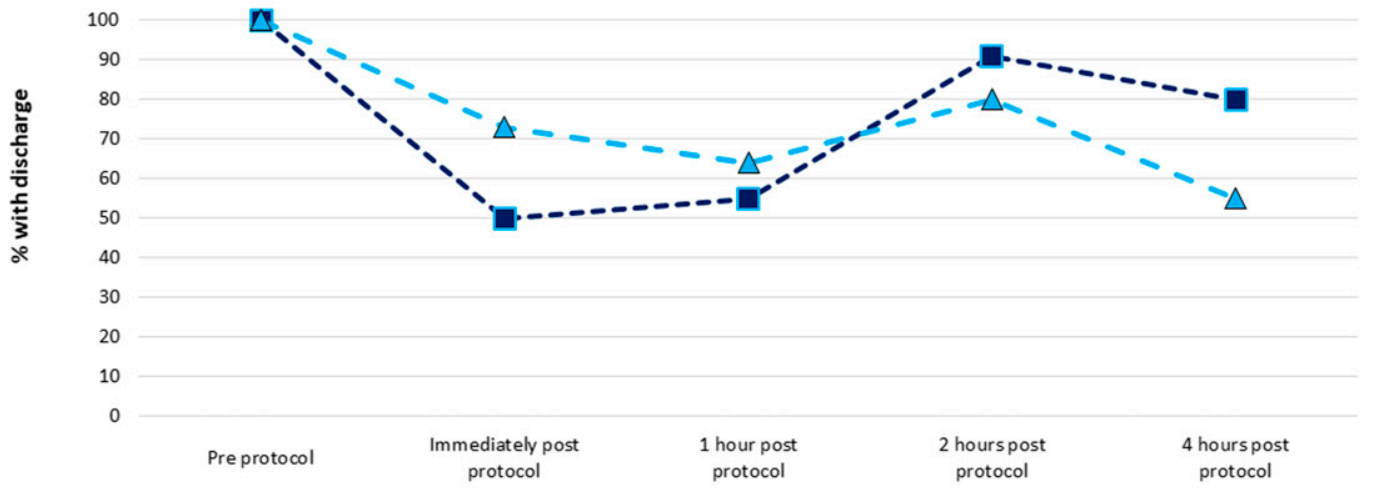

Total children with data - Washed with water Total children with data - Washed with soap $\%$ with discharge - Washed with water $\%$ with discharge - Washed with soap

14
11
100
100

14
11
50
73

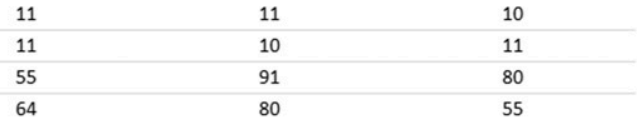

FIGURE 2. Presence of ocular and nasal discharge at five time points for children whose faces were washed with water or water and soap, among those with ocular (Graph A) or nasal (Graph B) discharge present on faces before washing. This figure appears in color at www.ajtmh.org. 
single other study of this type. ${ }^{9}$ This suggests we are most likely to interrupt transmission if face washing with soap is promoted at regular intervals throughout the day, rather than "daily," "twice a day," or "as frequently as necessary," as some programs currently recommend. An outstanding question is whether face washing with soap was more effective because of a property of the soap itself (i.e., friction) or because soap use caused more thorough washing (i.e., additional rinsing to remove soap). Given that we asked people to wash their faces in the same way regardless of soap use, our results suggest that the precise mechanism may not be important as it is likely to be difficult to achieve more thorough washing with water alone.

Our results suggest that washing with water and wiping with a hand might be insufficient to remove $C$. trachomatis from the faces of children in two-thirds of cases, whereas washing with soap may be more effective, although our sample of $C$. trachomatis-positive children was small, despite all having TF/TI. These results are consistent with other published studies. ${ }^{10}$

The main limitations of this study were the small sample size, the nonrandom allocation of protocols, and the nonblinded nature of the intervention for researchers. The design was pragmatic, but might have introduced bias in terms of classifying the presence of discharge or consistency of the procedures (albeit we had clear standard operating procedures and training). Despite these limitations, our findings suggest that it may be pertinent to explicitly promote soap use during face washing in trachoma-endemic settings where the prevalence of ocular discharge is very high. A randomized controlled trial is needed to indicate whether face washing with soap and water can interrupt C. trachomatis transmission.

Received October 1, 2019. Accepted for publication November 26, 2019.

Published online February 10, 2020.

Note: Supplemental table appears at www.ajtmh.org.

Acknowledgments: We are extremely grateful to the field team for their hard work and dedication throughout the fieldwork, in particular we wish to thank Asanti Ahmed, Ewnetu Belay, Gadisa Deresa, Meseret Guye, Demitu Legesses, Muira Haji Mohammed, and Sadia Oumer. We also would like to thank all our study participants and their families. This study would not have been possible without the support of The Fred Hollows Foundation Ethiopia and the Oromia Regional Health Bureau.

Financial support: The study was funded by the Wellcome Trust (206275/Z/17/Z). A. C., A. V., O. S., G. D., M. A. A., A. L., D. M., A. B., and K. G. were supported by a grant awarded to M. J. B.

Authors' addresses: Alexandra Czerniewska, Adam Biran, Sandy Cairncross, and Katie Greenland, Department of Disease Control, London School of Hygiene and Tropical Medicine, London, United Kingdom, E-mails: alexandra.czerniewska@Ishtm.ac.uk, adam.biran@Ishtm.ac.uk, sandy.cairncross@Ishtm.ac.uk, and
katie.greenland@Ishtm.ac.uk. Aalbertus Versteeg, Anna Last, and Matthew J. Burton, Clinical Research Department, London School of Hygiene and Tropical Medicine, London, United Kingdom, E-mails: aalbertus.versteeg@Ishtm.ac.uk, anna.last@Ishtm.ac.uk, and matthew.burton@Ishtm.ac.uk. Oumer Shafi, Gebeyehu Dumessa, Muluadam Abraham Aga, and Michael Kirumba, The Fred Hollows Foundation, Addis Ababa, Ethiopia, E-mails: oshafi@hollows.org, gbekele@hollows.org, maga@hollows.org, and mkirumba@hollows.org. David MacLeod, Department of Infectious Disease Epidemiology, London School of Hygiene and Tropical Medicine, London, United Kingdom, E-mail: david.macleod@ Ishtm.ac.uk. Virginia Sarah, The Fred Hollows Foundation, London, United Kingdom, E-mail: vsarah@hollows.org. Sarity Dodson, The Fred Hollows Foundation, Sydney, Australia, E-mail: sdodson@ hollows.org. Nebiyu Negussu, Federal Ministry of Health, Addis Ababa, Ethiopia, E-mail: nebiyu_negussu@yahoo.com. Birhanu Kenate Sori, Oromia Regional Health Bureau, Addis Ababa, Ethiopia, E-mail: simboyt@gmail.com.

This is an open-access article distributed under the terms of the Creative Commons Attribution (CC-BY) License, which permits unrestricted use, distribution, and reproduction in any medium, provided the original author and source are credited.

\section{REFERENCES}

1. Taylor HR, Burton MJ, Haddad D, West S, Wright H, 2014. Trachoma Lancet 384: 2142-2152.

2. Gower EW et al., 2006. Chlamydial positivity of nasal discharge at baseline is associated with ocular chlamydial positivity 2 months following azithromycin treatment. Invest Ophthalmol Vis Sci 47: 4767-4771.

3. Stocks ME, Ogden S, Haddad D, Addiss DG, McGuire C, Freeman MC, 2014. Effect of water, sanitation, and hygiene on the prevention of trachoma: a systematic review and meta-analysis. PLoS Med 11: e1001605.

4. Ejere HO, Alhassan MB, Rabiu M, 2015. Face washing promotion for preventing active trachoma. Cochrane Database Syst Rev 2: CD003659.

5. WHO, 2004. WHO simplified trachoma grading system. Community Eye Health 17: 68.

6. Last A et al., 2019. Detecting extra-ocular Chlamydia trachomatis in a trachoma-endemic community in Ethiopia: identifying potential routes of transmission. Under Review at PLoS Neglected Tropical Diseases.

7. West SK, Ansah D, Munoz B, Funga N, Mkocha H, 2017. The "F" in SAFE: reliability of assessing clean faces for trachoma control in the field. PLoS Negl Trop Dis 11: e0006019.

8. Butcher R, Houghton J, Derrick T, Ramadhani A, Herrera B, Last AR, Massae PA, Burton MJ, Holland MJ, Roberts Ch, 2017. Reduced-cost Chlamydia trachomatis-specific multiplex realtime PCR diagnostic assay evaluated for ocular swabs and use by trachoma research programmes. J Microbiol Methods 139: 95-102.

9. King JD, Ngondi J, Kasten J, Diallo MO, Zhu H, Cromwell EA, Emerson PM, 2011. Randomised trial of face-washing to develop a standard definition of a clean face for monitoring trachoma control programmes. Trans $R$ Soc Trop Med Hyg 105: 7-16.

10. Ramadhani AM, Derrick T, Macleod D, Holland MJ, Burton MJ, 2016. The relationship between active trachoma and ocular Chlamydia trachomatis infection before and after mass antibiotic treatment. PLOS Negl Trop Dis 10: e0005080. 